\title{
Short acquisition time PET quantification using MRI-based pharmacokinetic parameter synthesis
}

\author{
Catherine J Scott ${ }^{1}$, Jieqing Jiao ${ }^{1}$, M. Jorge Cardoso ${ }^{1}$, Andrew Melbourne ${ }^{1}$, \\ Enrico De Vita ${ }^{2,3}$, David L Thomas ${ }^{1,2,4}$, Ninon Burgos ${ }^{1,5}$, Pawel Markiewicz ${ }^{1}$, \\ Jonathan M Schott ${ }^{6}$, Brian F Hutton ${ }^{7,8}$, and Sébastien Ourselin ${ }^{1,6}$ \\ ${ }^{1}$ Translational Imaging Group, CMIC, University College London, London, UK \\ ${ }^{2}$ Neuroradiological Academic Unit, UCL Institute of Neurology, London, UK \\ ${ }^{3}$ Lysholm Department of Neuroradiology, National Hospital for Neurology and \\ Neurosurgery, UCL Hospitals Foundation Trust, London, UK \\ ${ }^{4}$ Leonard Wolfson Experimental Neurology Centre, UCL Institute of Neurology \\ ${ }^{5}$ Inria Paris, Aramis project-team, Sorbonne Universités, UPMC Univ Paris 06, \\ Inserm, CNRS, Institut du Cerveau et la Moelle épinière (ICM) - Pitié-Salpêtrière \\ Hospital, Paris, France \\ ${ }^{6}$ Dementia Research Centre, Institute of Neurology, University College London, UK \\ ${ }^{7}$ Institute of Nuclear Medicine, University College London, London, UK \\ ${ }^{8}$ Centre for Medical Radiation Physics, University of Wollongong, NSW, Australia
}

\begin{abstract}
Positron Emission Tomography (PET) with pharmacokinetic $(\mathrm{PK})$ modelling is a quantitative molecular imaging technique, however the long data acquisition time is prohibitive in clinical practice. An approach has been proposed to incorporate blood flow information from Arterial Spin Labelling (ASL) Magnetic Resonance Imaging (MRI) into PET PK modelling to reduce the acquisition time. This requires the conversion of cerebral blood flow $(\mathrm{CBF})$ maps, measured by ASL, into the relative tracer delivery parameter $\left(R_{1}\right)$ used in the PET PK model. This was performed regionally using linear regression between population $R_{1}$ and ASL values. In this paper we propose a novel technique to synthesise $R_{1}$ maps from ASL data using a database with both $R_{1}$ and CBF maps. The local similarity between the candidate ASL image and those in the database is used to weight the propagation of $R_{1}$ values to obtain the optimal patient specific $R_{1}$ map. Structural MRI data is also included to provide information within common regions of artefact in ASL data. This methodology is compared to the linear regression technique using leave one out analysis on 32 subjects. The proposed method significantly improves regional $R_{1}$ estimation $(p<0.001)$, reducing the error in the pharmacokinetic modelling. Furthermore, it allows this technique to be extended to a voxel level, increasing the clinical utility of the images.
\end{abstract}

\section{Introduction}

Positron Emission Tomography (PET) is a powerful molecular imaging technique which can be used to quantify the density of a target of interest in vivo, using a targeted radiotracer. However, full quantification requires a long, dynamic image 
acquisition to cover the delivery, binding and washout of the tracer, to facilitate PK modelling and the extraction of parameters of interest. Since an acquisition time of 60 minutes or more is not clinically feasible, instead a single static 10 minute acquisition is performed and the standardised uptake value ratio (SUVR) is calculated to estimate the relative uptake of the tracer. However, since this estimate is derived from a single time point, variations in blood flow, which influence the delivery and washout of the tracer, cannot be accounted for. Consequently SUVR measures become biased, confounding results in longitudinal studies where, in conditions such as Alzheimer's Disease (AD), there will be both changes in blood flow and the abundance of the biological target [1].

A framework to perform PK modelling on 30 minutes of PET data by incorporating blood flow information from simultaneously acquired MRI was proposed in [2]. This approach halves the acquisition time by assuming that the relationship between cerebral blood flow (CBF) measured using arterial spin labelling (ASL) MRI and the PET tracer delivery parameter $R_{1}$ can be approximated using a global linear regression derived from regional average values. However, image artefacts, limitations in image acquisition and assumptions in the ASL model used to calculate CBF mean that the errors within the CBF estimates vary across the brain. This will alter the relationship between ASL-CBF and PET tracer delivery [3]. Furthermore, due to low SNR and the resulting noise in the CBF estimates, performing linear regression for each region is non-trivial.

In this paper we propose to synthesise PET tracer delivery $R_{1}$ maps from ASL-CBF maps and structural T1 data using information propagation from a database of subjects with ASL-CBF, T1 and PET- $R_{1}$ data. Local similarity between the unseen candidate data and the database is used to propagate database PET- $R_{1}$ information into the candidate subject space. The use of a local similarity metric ensures that small-scale variation can be accounted for, whilst the use of a multi-modal database ensures that the method is robust to artefacts and uncertainties in the CBF estimation. This allows the technique to be extended to voxel-wise analysis which is required to detect local changes which differentiate between forms of disease and allow early diagnosis.

The methodology was evaluated in 32 subjects participating in a study of ageing/preclinical $\mathrm{AD}$ where the tracer target was amyloid- $\beta$, as quantified by the binding potential $\left(B P_{N D}\right)$. Our approach gives a significantly improved estimation of $R_{1}$ on both regional and voxel-wise scales compared to [2]. This translates into significantly lower errors in $B P_{N D}$ estimates, such that amyloid burden can be accurately quantified using a single 30 minute PET/MRI acquisition.

\section{Methods}

\subsection{MRI-PET database construction and $R_{1}$ synthesis}

For each of the $N$ subjects forming the database, the MRI data (including structural T1 and ASL-CBF) are affinely registered into PET- $R_{1}$ space. The input database thus consists of ASL-CBF and T1 data, and the output database contains the corresponding subject PET- $R_{1}$ maps, as shown in Fig. 1 . For $R_{1}$-map 
synthesis, the multi-contrast approach from [4] is used. The ASL-T1 pairs in the input database are registered to the candidate data using an affine registration to initialise the non-rigid registration [5]. The convolution based local normalised cross correlation (LNCC) is then calculated between each registered ASL-T1 pair in the database and the candidate ASL-T1 pair. This local similarity metric is then summed across the 2 channels, and is used to weight the contribution of each propagated $R_{1}$-map from the output database to the synthetic $R_{1}$-map [6].

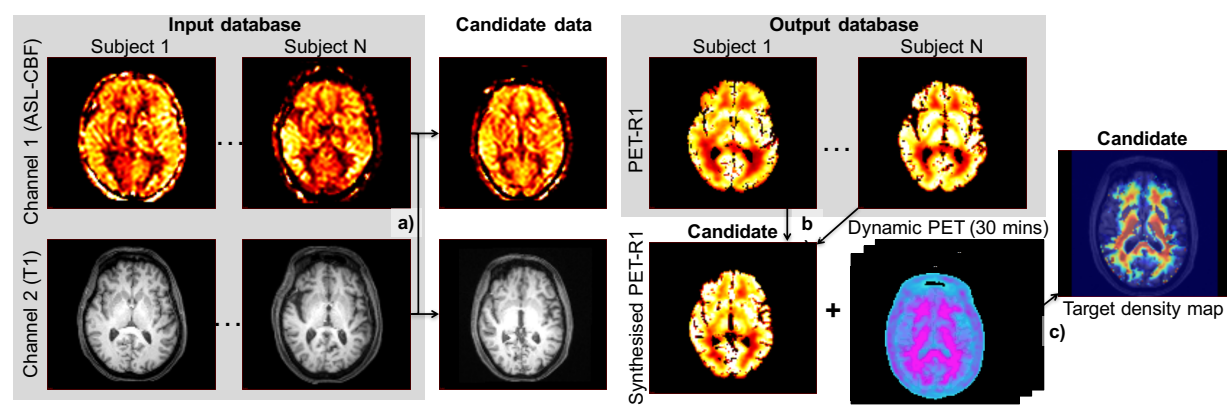

Fig. 1: a) Multi-channel registration transforms input database images into the candidate subject space. The local similarity between the images is calculated, b) and is used to weight the propagation of $R_{1}$ information from the output database into candidate space. c) The synthesised $R_{1}$ map is combined with dynamic PET data and PK modelling is used to derive the target density map.

\subsection{PET quantification using the SRTM}

The simplified reference tissue model (SRTM) [7] is used to quantify the PET data, using the linearised formulation as in (1), where a set of basis functions for $C_{R}(t) \otimes e^{-\theta t}$ are pre-calculated over a physiologically plausible range of $\theta[8]$. The tracer-target interaction is modelled using one tissue compartment where the tracer concentration in the reference region $C_{R}(t)$ is the input function, and the target tissue concentration is $C_{T}(t)$. The model contains 3 parameters, $R_{1}$ (the rate of delivery in the target tissue relative to reference tissue), $k_{2}$ (the transfer rate constant from target tissue to blood), and the parameter of interest $B P_{N D}$ (the binding potential which is related to target density and consequently amyloid- $\beta$ burden). The parameters are estimated using curve fitting to $C_{R}(t)$ and $C_{T}(t)$ measured from $t=0$ at tracer injection over a sufficient duration.

$$
\begin{array}{r}
C_{T}(t)=R_{1} C_{R}(t)+\phi C_{R}(t) \otimes e^{-\theta t} \\
\text { where } \phi=k_{2}-R_{1} k_{2} /\left(1+B P_{N D}\right), \quad \theta=k_{2} /\left(1+B P_{N D}\right)
\end{array}
$$


Cerebellar grey matter is used as the reference region for $C_{R}(t)$ as it is assumed to be devoid of amyloid- $\beta$ [9]. $B P_{N D}, R_{1}$ and $k_{2}$ were calculated from dynamic PET data acquired from $t=0$ to $t=60(0: 60)$ minutes as the gold standard.

\subsection{CBF estimation from ASL MRI}

Cerebral blood flow (CBF) maps were estimated from pseudo-continuous ASL (PCASL) data and saturation recovery images at 3 recovery times $(1,2,4 \mathrm{~s})[10]$. The proton density, $S_{0}$, was estimated by fitting the saturation recovery images for $\left[\mathrm{T} 1, S_{0}\right]$. The parameter values used were $0.9 \mathrm{ml} / \mathrm{g}$ for the plasma/tissue partition coefficient, $1650 \mathrm{~ms}$ for the blood T1, and 0.85 for labelling efficiency.

\subsection{SRTM with incomplete PET scan and CBF}

Population-based extrapolation of reference input $\boldsymbol{C}_{\boldsymbol{R}}(\boldsymbol{t})$. To calculate the convolution term in (1), $C_{R}(t)$ for $t \in\left[0, t_{e}\right]$, where $t=0$ at injection and $t=t_{e}$ at the end of the acquisition, is required. When the PET acquisition time is reduced to $t \in\left[t_{s}, t_{e}\right], C_{R}(t)$ for $t \in\left[0, t_{s}\right]$ must be estimated. This is performed as in [2], where a set of subjects with $C_{R}(t)$ for $t \in\left[0, t_{e}\right]$ are used to generate a mean population $C_{R}(t)$, which is then scaled to the available $C_{R}(t) t \in\left[t_{s}, t_{e}\right]$ to estimate a subject specific $C_{R}(t)$ for $t \in\left[0, t_{e}\right]$.

SRTM with CBF-derived $\boldsymbol{R}_{1}$ and extrapolated $\boldsymbol{C}_{\boldsymbol{R}}(\boldsymbol{t})$. (1) is re-written $C_{T}(t)-R_{1} C_{R}(t)=\phi C_{R}(t) \otimes e^{-\theta t}$, to group the measured terms $\left(C_{T}(t), C_{R}(t)\right.$ for $t \in\left[t_{s}, t_{e}\right]$ ) with the CBF-derived $R_{1}$. Basis functions were generated using a range of $\theta$ values and the extrapolated $C_{R}$ for $t \in\left[0, t_{e}\right]$, and $\phi$ is estimated using a least squares fit of the data. The combination of $\phi$ and $\theta$ which minimise the least squares error in the fit are then used to calculate $B P_{N D}$ and $k_{2}$.

\section{Experiments and Results}

Data. Imaging data were collected from 32 cognitively normal subjects participating in Insight 46, a neuroimaging sub-study of the MRC National Survey of Health and Development, who underwent simultaneous PET and multimodal MRI on a Siemens Biograph mMR 3T PET/MRI scanner [11]. List mode PET data were acquired for 60 minutes following intravenous injection of $\left[{ }^{18} \mathrm{~F}\right]$ florbetapir, an amyloid- $\beta$ targeting radiotracer. Structural 3D T1- and T2-weighted MR images were used to estimate the attenuation map [4]. Dynamic PET data were binned into $15 s \times 4,30 s \times 8,60 s \times 9,180 s \times 2,300 s \times 8$ time frames, and reconstructed into $2 \times 2 \times 2 \mathrm{~mm}$ voxels using in-house software with corrections for dead-time, attenuation, scatter, randoms and normalisation [12]. PCASL ASL data were acquired using a $3 \mathrm{D}$ GRASE readout at $3.75 \times 3.75 \times 4 \mathrm{~mm}$ and reconstructed to $1.88 \times 1.88 \times 4 \mathrm{~mm}$ resolution voxels. 10 control-label pairs were acquired with a pulse duration and post labelling delay of $1800 \mathrm{~ms}$. For regional analysis and reference region delineation, T1 data were parcellated [6] and propagated into PET space. 
Validation. Leave one out analysis was used such that $N-1$ subjects were used in the database, for linear regression between ASL-CBF and PET- $R_{1}$, and for extrapolation of the reference region $C_{R}$. The comparison of methods was performed for both $R_{1}$ and $B P_{N D}$ estimation using the mean absolute error (MAE $=1 / v \sum_{v}\left|I_{v}^{e s t}-I_{v}^{G S}\right|$ where $I$ is intensity, $v$ is the number of voxels or regions, $G S$ is the gold standard and est is the estimated value). Statistical tests on MAE were performed using the paired Wilcoxon signed rank test. For SUVR estimation, 50:60 minutes of PET data were normalised by the mean reference region intensity, as this time window gave the lowest error compared to the gold standard. For PK modelling using reduced acquisition time, 30:60 minutes of PET data were used. PK modelling using 30:60 minutes of data and the gold standard 'true' $R_{1}$ was calculated as the ideal case where $R_{1}$ is estimated exactly.

\subsection{Regional Analysis of $R_{1}$ and $B P_{N D}$ estimation}

Regional analysis was performed by averaging across 17 regions: 6 cortical grey matter regions, accumbens, amygdala, brainstem, caudate, cerebral white matter, hippocampus, pallidum, putamen, thalamus, cerebellar white matter and cerebellar grey (reference region), with right and left hemispheres combined.
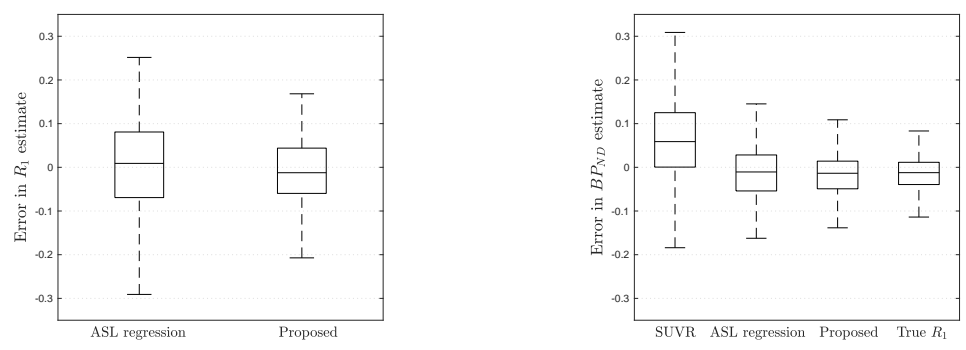

Fig. 2: Regional errors in $R_{1}$ and $B P_{N D}$, outliers excluded for visualisation.

$\boldsymbol{R}_{1}$ estimation accuracy. Fig. 2 left shows the errors in the $R_{1}$ estimation compared to the gold standard, demonstrating that the proposed synthesis method gives improved estimates over those obtained using linear regression on the ASL values directly. For the proposed synthesised $R_{1}$ the MAE is significantly reduced $(p<0.001$ ), from 0.08668 using the ASL regression method to 0.06326 using the synthesised $R_{1}$. This reflects the flexibility of the synthesis method to account for regional differences in the relationship between CBF and $R_{1}$.

Binding potential $\left(\boldsymbol{B} \boldsymbol{P}_{\boldsymbol{N}}\right)$ estimation accuracy. The errors in the estimation of $B P_{N D}$ using fixed $R_{1}$ values and 30 minutes of PET data are shown 
in Fig. 2 right. This illustrates the known positive bias in the SUVR compared to the gold standard and the reduction in errors when PK modelling is used. For the fit using the proposed synthesised $R_{1}$ map, the error is reduced compared to linear regression on the ASL values. This is not reflected in the MAE where the proposed method and the ASL regression method achieve an MAE of 0.07495 and 0.07501 respectively. However, the distributions are statistically significantly different $(p=0.02)$. Both $R_{1}$ estimation techniques perform significantly better than SUVR $(p<0.001)$, and produce similar errors to those obtained using the gold standard $R_{1}(\mathrm{MAE}=0.06145)$.

\subsection{Voxel-wise Analysis of $R_{1}$ and $B P_{N D}$ estimation}

Voxel-wise analysis was performed on all voxels contained within the subject brain mask. MAE was calculated for each subject then averaged across subjects.

$\boldsymbol{R}_{1}$ estimation accuracy. Fig. 3 left shows the reduction in $R_{1}$ estimate error using $R_{1}$ synthesis compared to the ASL regression technique. This is reflected in the significantly reduced MAE, which is 0.21175 for ASL regression as opposed to 0.15979 for $R_{1}$ synthesis $(p<0.001)$. Fig. 3 right shows that the synthesised $R_{1}$ maps are better able to capture local differences in tracer delivery than those generated using ASL regression. This is due to the shallow slope obtained from the linear regression which reduces the range of the ASL-CBF maps.
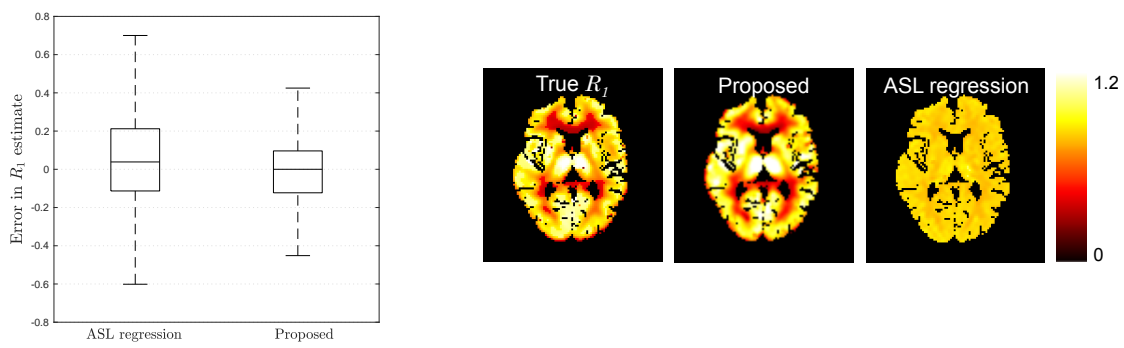

Fig. 3: Voxel-wise $R_{1}$ : Left- errors (outliers not shown), Right- example maps

Binding potential $\left(\boldsymbol{B} \boldsymbol{P}_{\boldsymbol{N} D}\right)$ estimation accuracy. Fig. 4 left shows the mean error in $B P_{N D}$ estimates compared to the gold standard and as for the regional analysis, SUVR shows a positive bias. The ASL regression technique also has a small positive bias which is not present for the synthesised and true $R_{1}$ values. Again, the MAE is significantly lower for the synthesised $R_{1}$ compared to the ASL-regression method. The mean errors using the synthesised $R_{1}$ and gold standard $R_{1}$ appear similarly distributed, however the MAE is significantly lower 
when using the gold standard, where MAE $=0.1593$ compared to 0.17687 for synthesised $R_{1}(p<0.001)$. Fig. 4 right demonstrates that the proposed method is a good approximation of the $B P_{N D}$ using the gold standard $R_{1}$, however all of the PK modelling techniques using 30 minutes of data show corruption due to the noise in the PET data on a voxel level which is enhanced when halving the acquisition time.
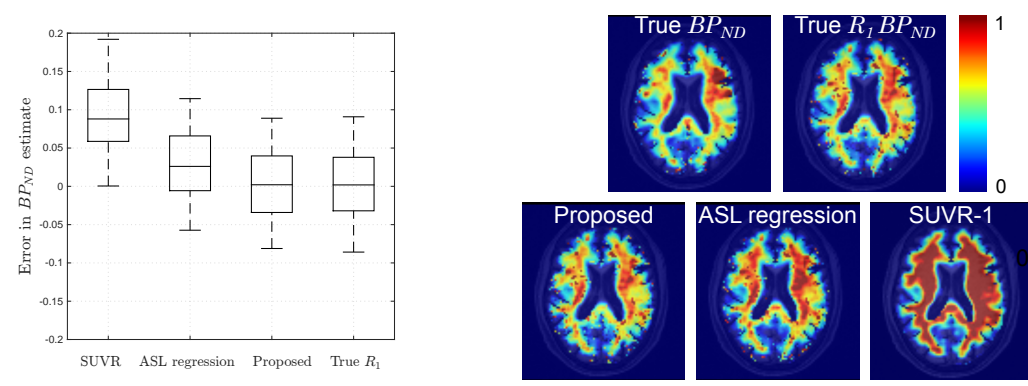

Fig. 4: Voxel-wise $B P_{N D}$ results: Left- Errors in $B P_{N D}$ (outliers excluded for display) Right- Example $B P_{N D}$ maps overlaid on T1 images for visualisation

\section{Discussion and Conclusion}

In this paper we present a novel methodology to synthesise PET tracer delivery, $R_{1}$, maps from a database of ASL-CBF and T1 images to significantly improve the quantification of PET data using a clinically feasible acquisition time. The results were compared to a technique proposed in [2], where $R_{1}$ values were derived directly from the ASL data by linear regression of a population of subjects with ASL-CBF and $R_{1}$ maps. For regional analysis, the proposed synthesised $R_{1}$ estimation performed significantly better than the ASL regression method, and the performance in the estimation of $B P_{N D}$ following PK modelling on 30:60 minutes of PET data was also significantly better. The analysis was further extended to a voxel level where the higher accuracy and resolution of the synthesised $R_{1}$ map led to a significant improvement in $B P_{N D}$ estimation.

This work demonstrates that $B P_{N D}$ quantification is possible on a voxel level for a 30 minute PET/MR acquisition. However, fitting noisy voxel-wise data is susceptible to errors which are increased for reduced acquisition times. In future work we will apply the SRTM with a spatial constraint to improve the stability of voxel-wise fitting [13]. Furthermore, we will consider that the images within the database are imperfect, i.e. the ASL-CBF maps are susceptible to artefacts, and the $R_{1}$ maps are voxel-wise fits to noise corrupted data. This information may be used to modify the similarity estimation between the candidate and the database to increase robustness in the presence of artefacts. 
Acknowledgements This work was supported by the EPSRC UCL Centre for Doctoral Training in Medical Imaging (EP/L016478/1), UCL Leonard Wolfson Experimental Neurology Centre (PR/ylr/18575), EPSRC (EP/H046410/1, $\mathrm{EP} / \mathrm{J} 020990 / 1, \mathrm{EP} / \mathrm{K} 005278)$, MRC (MR/J01107X/1), NIHR UCLH Biomedical Research Centre (inc. High Impact Initiative, BW.mn.BRC10269). Insight 1946 receives funding from Alzheimer's Research UK (ARUK-PG2014-1946), MRC Dementia Platform UK (CSUB19166) and The Wolfson Foundation, and support from Avid Radiopharmaceuticals, a wholly owned subsidiary of Eli Lilly. We are grateful to the Insight 46 participants for their involvement in this study.

\section{References}

1. Cselényi, Z., Farde, L.: Quantification of blood flow-dependent component in estimates of beta-amyloid load obtained using quasi-steady-state standardized uptake value ratio. J Cereb Blood Flow Metab 35(9) (2015) 1-9

2. Scott, C.J., Jiao, J., Melbourne, A., et al: ASL-incorporated Pharmacokinetic Modelling of PET Data With Reduced Acquisition Time: Application to Amyloid Imaging. Med Image Comput Comput Assist Interv, LNCS 9902 (2016) 406-413

3. Fan, A.P., Jahanian, H., Holdsworth, S.J., et al: Comparison of cerebral blood flow measurement with [15O]-water PET and arterial spin labeling magnetic resonance imaging: A systematic review. J Cereb Blood Flow Metab 36(5) (2016) 842-861

4. Burgos, N., Cardoso, M.J., Thielemans, K., et al.: Multi-contrast Attenuation Map Synthesis for PET/MR Scanners: Assessment on FDG and Florbetapir PET Tracers. Eur J of Nuc Med and Mol Imaging 42(9) (2015) 1447-1458

5. Modat, M., Ridgway, G.R., et al: Fast free-form deformation using graphics processing units. Comput Methods Programs Biomed 98(3) (2010) 278-284

6. Cardoso, M.J., Modat, M., Wolz, R., et al: Geodesic information flows: spatiallyvariant graphs and their application to segmentation and fusion. IEEE TMI 34(9) (2015) 1976-1988

7. Lammertsma, A., Hume, S.: Simplified reference tissue model for PET receptor studies. NeuroImage 158(4) (1996) 153-158

8. Gunn, R.N., Lammertsma, A.A., Hume, S.P., Cunningham, V.J.: Parametric imaging of ligand-receptor binding in PET using a simplified reference region model. NeuroImage 6(4) (1997) 279-287

9. Klunk, W.E., Engler, H., Nordberg, A., et al: Imaging Brain Amyloid in Alzheimer's Disease with Pittsburgh Compound-B. Ann. Neurol 55(3) (2004) 306-319

10. Melbourne, A., Toussaint, N., Owen, D., et al: Niftyfit: a software package for multiparametric model-fitting of 4D magnetic resonance imaging data. Neuroinformatics 14(3) (2016) 319-337

11. Lane, C.A., Parker, T.D., Cash, D.M., et al.: Study protocol: Insight 46 a neuroscience sub-study of the MRC National Survey of Health and Development. BMC Neurology 17(1) (2017) 75

12. Markiewicz, P.J., Thielemans, K., Schott, J., et al: Rapid processing of PET listmode data for efficient uncertainty estimation. Phys Med Bio 61(3) (2016) 322-336

13. Zhou, Y., Resnick, S.M., Ye, W., et al: Using a reference tissue model with spatial constraint to quantify [11C]Pittsburgh compound B PET for early diagnosis of Alzheimer's disease. NeuroImage 36(2) (2007) 298-312 